\title{
Thresholdless behavior and linearity of the optically induced metallization of $\mathrm{NbO}_{2}$
}

\author{
J. Kenji Clark, Ya-Lun Ho, Hiroaki Matsui, Hitoshi Tabata, and Jean-Jacques Delaunay* \\ School of Engineering, The University of Tokyo, Tokyo, Japan
}

(Received 17 March 2019; revised manuscript received 16 October 2019; published 11 December 2019)

\begin{abstract}
The optical excitation of $\mathrm{NbO}_{2}$ is known to induce a transient metallicity that partially recovers on a subpicosecond speed and only fully recovers after several nanoseconds. Here, through low-fluence pump-probe spectroscopy, the short-lived and long-lived components of $\mathrm{NbO}_{2}$ 's optically induced modulation are found to be linearly dependent on pump fluence in the low-fluence regime. Through an in-depth analysis, the short-lived and long-lived modulations are found to be caused by photocarrier-induced refractive index changes and photothermally induced changes in the lattice temperature of $\mathrm{NbO}_{2}$, respectively, and not an insulator-metal transition.
\end{abstract}

DOI: 10.1103/PhysRevResearch.1.033168

\section{INTRODUCTION}

In recent years, interest in all-optical computing devices, in particular, all-optical modulators that modulate the intensity of an optical signal by means of another optical signal, has grown [1]. Using nonlinear effects in silicon waveguides [2], photonic crystals [3,4], and graphene [5], all-optical modulators have been demonstrated; however, their reliance on weak nonlinear phenomena necessitates large device sizes that make them unattractive for most applications.

Highly correlated oxide materials, such as $\mathrm{VO}_{2}$ and $\mathrm{NbO}_{2}$, have been proposed as potential materials for nanoscale alloptical modulators. These materials undergo an insulatormetal transition (IMT) accompanied by a crystal phase transition (CPT) when their temperature is raised above an IMT temperature [6,7]. Upon undergoing an IMT, the optical properties of such materials also change drastically, and this has enabled many groups to demonstrate high-performance electro-optic [8,9] and thermo-optic [10] modulators with nanoscale sizes.

In recent years, it has also been shown that the IMT of $\mathrm{VO}_{2}$ can be induced optically through excitation by a femtosecond laser pulse. Whether or not this optically induced IMT is the result of a Mott-Hubbard transition or Peierls transition has been and continues to be the focus of a large number of research articles [11-17]. Yet despite this large amount of work, the exact nature of the IMT is still not fully understood [18].

A thorough understanding of the mechanism of $\mathrm{VO}_{2}$ 's IMT is complicated by the low temperature $(340 \mathrm{~K})$ of the IMT [19]. To isolate the optically and thermally induced phenomena, interest has grown in another closely related material, $\mathrm{NbO}_{2}$, whose IMT is at a much higher temperature $(1081 \mathrm{~K})$. In the first study of the optically induced IMT of $\mathrm{NbO}_{2}, \mathrm{NbO}_{2}$

\footnotetext{
${ }^{*}$ Corresponding author: jean@mech.t.u-tokyo.ac.jp

Published by the American Physical Society under the terms of the Creative Commons Attribution 4.0 International license. Further distribution of this work must maintain attribution to the author(s) and the published article's title, journal citation, and DOI.
}

was pumped by a femtosecond laser pulse at $800 \mathrm{~nm}$ and the transient change in the reflectance at $800 \mathrm{~nm}$ was measured [19]. The $\mathrm{NbO}_{2}$ exhibited a transient decrease in reflectance with a recovery described by three time constants: an ultrafast fs response attributed to electron-electron scattering of photoexcited carriers, a short-lived ps response attributed to the recovery of grains of $\mathrm{NbO}_{2}$ that undergo a purely electronic IMT, and a long-lived ns response attributed to the recovery of grains of $\mathrm{NbO}_{2}$ that undergo both an electronic IMT and a structural CPT.

A later study of the optically induced IMT of $\mathrm{NbO}_{2}$ studied the transient change in the terahertz conductance of $\mathrm{NbO}_{2}$ after excitation by an $800-\mathrm{nm}$ pump laser pulse [20]. The terahertz conductivity of $\mathrm{NbO}_{2}$ was shown to exhibit a transient increase governed by the same three timescales. Nonlinearities in the long-lived response at 10 and $17.5 \mathrm{~mJ} / \mathrm{cm}^{2}$ were attributed to the IMT being induced by photocarrier excitation and photothermal excitation, respectively.

There are still several aspects of the $\mathrm{NbO}_{2}$ pump-probe response that are not well understood. First, it is unclear whether or not the short-lived ps recovery and long-lived ns recovery are the result of an IMT, or if they are the result of photoexcited carriers in the $\mathrm{NbO}_{2}$. In addition, it is not yet clear that $\mathrm{NbO}_{2}$ can exhibit a short-lived modulation without the additional long-lived modulation, which is critical for $\mathrm{NbO}_{2}$ to be applied to ultrahigh-speed all-optical modulators.

In this paper, we study the transient modulation of the transmittance and reflectance of an $\mathrm{NbO}_{2}$ thin film at $1550 \mathrm{~nm}$ ( $C$ band) after excitation by a 766-nm femtosecond laser pulse. We examine the short-lived and long-lived responses in the low-fluence regime and demonstrate that both responses scale linearly with fluence with no evidence of an IMT threshold. Through an analysis of the photothermally induced temperature change, the long-lived modulation is found to be a result of the photothermal excitation of $\mathrm{NbO}_{2}$. Additionally, an in-depth theoretical analysis that accounts for the BursteinMoss effect, band shrinkage effect, and free carrier absorption effect shows that the short-lived modulation results from photoexcited carrier-induced changes in the optical properties of $\mathrm{NbO}_{2}$. 

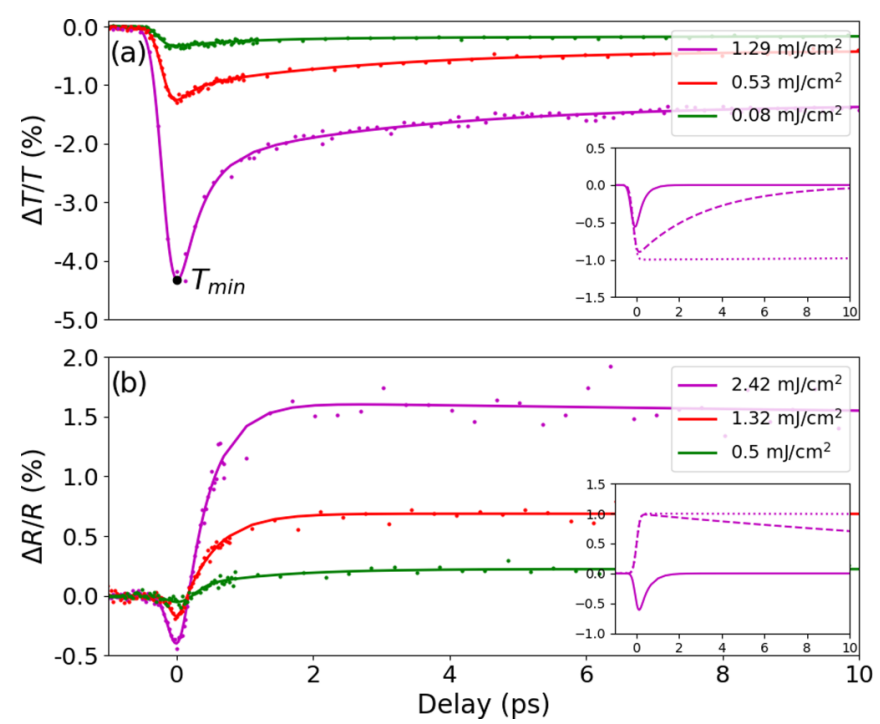

FIG. 1. Relative change in (a) transmittance and (b) reflectance (b) of the $\mathrm{NbO}_{2}$ to the $1550-\mathrm{nm}$ probe pulse plotted against the pump-probe delay. Insets show the components of the pump-probe response.

\section{RESULTS}

To study the femtosecond response of $\mathrm{NbO}_{2}$, a $60-\mathrm{nm}$ $\mathrm{NbO}_{2}$ thin film was fabricated on a 0.5 -mm-thick $\mathrm{Al}_{2} \mathrm{O}_{3}$ $c$-plane (0001) substrate using pulsed laser deposition (PLD) and the transient modulation after photoexcitation was measured. Full details of the fabrication, characterization, and measurement of the $\mathrm{NbO}_{2}$ thin film are given in the Supplemental Material [21].

The modulation in the transmittance of the $\mathrm{NbO}_{2}$ film at $1550 \mathrm{~nm}$ upon excitation by the pump pulse is shown in Fig. 1(a). The response is similar to that seen when both the pump and probe pulses have a wavelength of $800 \mathrm{~nm}$ [19]. An initial strong decrease in the transmittance that recovers after several ps is followed by a long-lived decrease in the transmittance that recovers in several hundred ps to several ns. To better understand the dynamics of the modulation, the response was fit to a three-exponential decay curve [19],

$$
\frac{\Delta T}{T}=\left[1+\operatorname{erf}\left(\frac{t-t_{0}}{\tau_{p}}\right)\right]\left(A_{1} e^{\frac{t-t_{0}}{\tau_{1}}}+A_{2} e^{\frac{t-t_{0}}{\tau_{2}}}+A_{3} e^{\frac{t-t_{0}}{\tau_{3}}}\right),
$$

where $t_{0}$ is the delay between the pump and probe pulses, $\tau_{p}$ is related to the laser pulse width and the response time of the material, and $\tau_{1}, \tau_{2}$, and $\tau_{3}$ are the time constants of the three decay rates. The components of the decay for the $1.29 \mathrm{~mJ} / \mathrm{cm}^{2}$ curve are shown in the inset of Fig. 1(a). There is an ultrafast decay with a $\tau_{1}$ of $324 \mathrm{fs}$, a short-lived decay with a $\tau_{2}$ of $3.24 \mathrm{ps}$, and a long-lived decay with a $\tau_{3}$ of $543 \mathrm{ps}$. As the pump fluence increases, the exact values of the decay time constants vary, but the three timescales were consistently observed.

The reflectance was subsequently modeled with Eq. (1). The ultrafast, short-lived, and long-lived modulations are all observed; however, whereas the short-lived and long-lived modulations correspond to decreases in the transmittance at

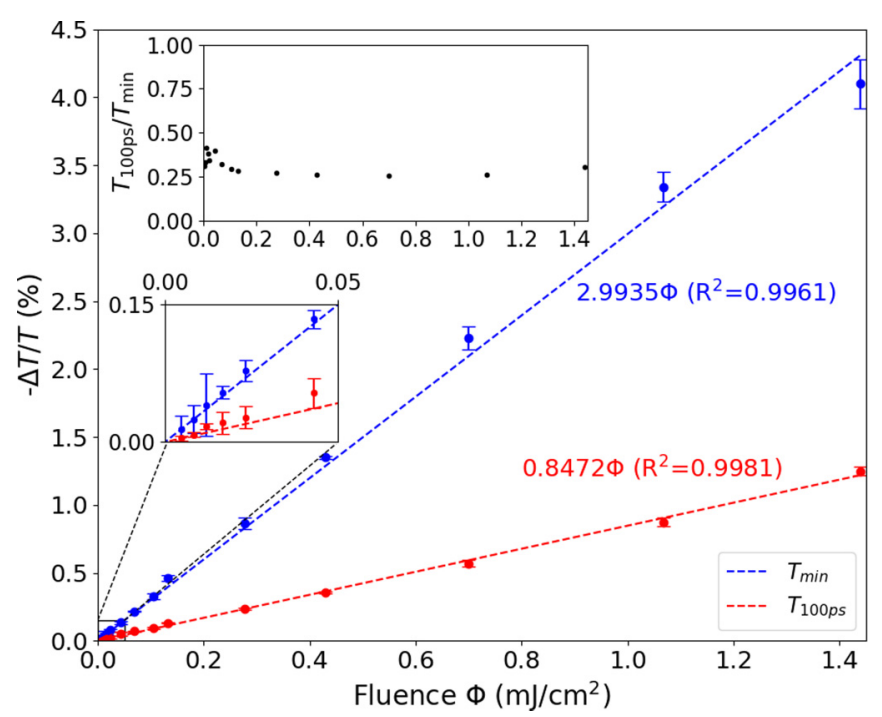

FIG. 2. Modulation in the $1550-\mathrm{nm}$ transmittance of the $\mathrm{NbO}_{2}$ at the minimum of transmittance (blue) and at a 100-ps delay (red). Center left inset: Low-fluence region. Top inset: Ratio of the modulation at $100 \mathrm{ps}$ and at the transmittance minimum.

$1550 \mathrm{~nm}$ and the reflectance at $800 \mathrm{~nm}$ [19], they represent increases in the reflectance at $1550 \mathrm{~nm}$.

Subsequently, the modulation in the transmittance for pump fluences between $4.6 \mu \mathrm{J} / \mathrm{cm}^{2}$ and $1.4 \mathrm{~mJ} / \mathrm{cm}^{2}$ was measured. The maximum transmittance modulation $\left[T_{\min }\right.$ in Fig. 1(a)] and the modulation at $100 \mathrm{ps}$ after the maximum modulation $\left(T_{100 \mathrm{ps}}\right)$ are shown in Fig. 2. $T_{\min }$ includes the combined effect of all three modulations (ultrafast, short lived, and long lived), whereas $T_{100 \mathrm{ps}}$ includes only the long-lived modulation. For the entire range of fluences studied, $T_{\min }$ and $T_{100 \mathrm{ps}}$ scale linearly with pump fluence, with no evidence of any threshold. Furthermore, the ratio of $T_{\min }$ to $T_{100 \mathrm{ps}}$, as shown in the upper inset of Fig. 2, was relatively constant (only fluctuating at very low fluences where the signal-tonoise ratio was poor).

\section{DISCUSSION}

These results contradict the idea that the long-lived modulation is due to an IMT. Based on the Mott-Hubbard description, an IMT occurs when the concentration of excited carriers in the conduction band reaches a threshold level at which they sufficiently screen the Coulomb interaction and cause the band gap of the insulator to collapse [18]. This results in a drastic increase in the metallic reflection of the material, and therefore, a nonlinearity in the fluence versus modulation curve should be expected. For this low-fluence regime, however, no such threshold exists for fluences as low as $4.6 \mu \mathrm{J} / \mathrm{cm}^{2}$.

It can also be shown that a nonlinearity and IMT onset cannot exist below $4.6 \mu \mathrm{J} / \mathrm{cm}^{2}$. By assuming that all of the absorbed pump light contributes to the excitation of photoexcited carriers, the photoexcited carrier concentration $N$ can be calculated as

$$
N=\frac{(1-R-T) \Phi_{\mathrm{pump}}}{t_{\mathrm{film}} E_{\mathrm{ph}}} .
$$




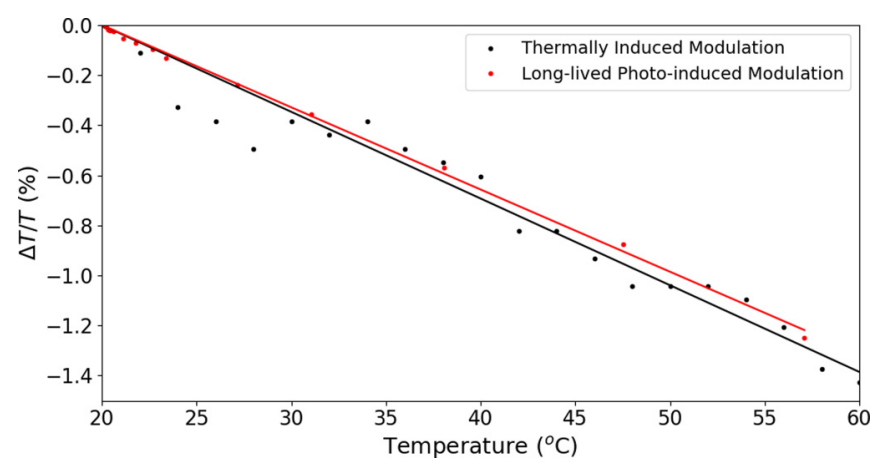

FIG. 3. Thermally induced modulation in $\mathrm{NbO}_{2}$ 's transmittance relative to room-temperature transmittance (black) compared to the long-lived modulation at different pump fluences (red).

Here, $\Phi_{\text {pump }}$ is the pump fluence, $t_{\text {film }}$ is the thickness of the $\mathrm{NbO}_{2}$ film $(60 \mathrm{~nm}), E_{\mathrm{ph}}$ is the photon energy of the pump (1.62 $\mathrm{eV}$ for a 766-nm pump), and $R$ and $T$ are the reflectance $(27.6 \%)$ and transmittance $(32.3 \%)$ of the sample at the pump wavelength. Using this equation, a pump fluence of $4.6 \mu \mathrm{J} / \mathrm{cm}^{2}$ would correspond to a photoexcited carrier density of only $1.18 \times 10^{18}$ carriers $/ \mathrm{cm}^{3}$. This is several orders of magnitude lower than the intrinsic carrier concentration of $\mathrm{NbO}_{2}$ just before the thermally induced IMT [22], and since a thermally induced IMT does not occur when the thermally excited carrier concentration reaches this concentration, the concentration must be insufficient to trigger an IMT.

The simplest explanation for the origin of the long-lived modulation is that it is the result of photothermally induced changes in the lattice temperature of $\mathrm{NbO}_{2}$ and the resulting thermo-optic changes in the optical properties of $\mathrm{NbO}_{2}$. When $\mathrm{NbO}_{2}$ absorbs the pump light, excited electrons and holes are generated, and as they relax and recombine, absorbed energy is released. Because $\mathrm{NbO}_{2}$ has an indirect band gap, this energy is primarily released in the form of phonons that raise the lattice temperature. The temperature change $\Delta T$ is given by

$$
\Delta T=\frac{(1-R-T) \Phi_{\text {pump }} M_{u}}{t_{\text {film }} \rho_{\mathrm{NbO}_{2}} C_{p}},
$$

where $M_{u}$ is the molar mass of $\mathrm{NbO}_{2}(124.9 \mathrm{~g} / \mathrm{mol}), \rho_{\mathrm{NbO}_{2}}$ is its density $\left(6.10 \mathrm{~g} / \mathrm{cm}^{3}\right), C_{p}$ is its heat capacity $(57.35 \mathrm{~J} / \mathrm{mol} \mathrm{K}$ [23]), and $\Phi_{\text {pump }}, t_{\text {film }}, R$, and $T$ are the same as above. The lattice temperature change induced for the fluence range measured in Fig. 2 was first calculated according to the equation above. $T_{100 \mathrm{ps}}$ was then plotted against the induced temperature change and compared to the thermally induced modulation in Fig. 3. The thermally induced modulation was calculated by measuring the transmittance of the $\mathrm{NbO}_{2}$ film at $1.55 \mu \mathrm{m}$ over a temperature range of $20-60^{\circ} \mathrm{C}$ and then calculating the modulation in the transmittance relative to the transmittance at $20^{\circ} \mathrm{C}$. Figure 3 shows a strong agreement between the measured thermally induced modulation and the long-lived photoinduced modulation, supporting the argument that the long-lived modulation is a result of the photothermally induced change in $\mathrm{NbO}_{2}$ 's lattice temperature. The recovery of the long-lived modulation, then, is simply due to the dissipation of the excess heat into the substrate.
The ps recovery time of the short-lived modulation suggests an origin related to photoexcited carriers in the $\mathrm{NbO}_{2}$. When electrons and holes are excited in a semiconductor material, three main phenomena occur that work to alter the electronic band structure and optical properties of the material. They are the Burstein-Moss effect, the band-gap shrinkage effect, and the free carrier absorption effect [24].

In the Burstein-Moss effect, also known as the band filling effect, the excitation of electrons from the valence band to the conduction band of a semiconductor results in a reduction of the absorption coefficient of the semiconductor near the band-gap energy [24]. This is because excited electrons occupy states in the conduction band and leave behind holes in the valence band, preventing other electrons from absorbing photons and being excited. The change in the absorption coefficient $\Delta \alpha$ can be modeled as

$$
\Delta \alpha(E)=\alpha_{0}(E)\left[f_{v}\left(E_{a}\right)-f_{c}\left(E_{b}\right)-1\right],
$$

where $\alpha_{0}$ is the original absorption coefficient and $f_{v}\left(E_{a}\right)$ and $f_{c}\left(E_{b}\right)$ are the electron occupation probabilities at an energy $E_{a}$ below the valence band maximum and an energy $E_{b}$ above the conduction band minimum, respectively. The calculations of $f_{v}$ and $f_{c}$ are elaborated on in the Supplemental Material [21]. $\alpha_{0}$ was determined from ellipsometric measurements of $\mathrm{NbO}_{2}$ 's refractive index. From the absorption coefficient, the change in the imaginary part of the refractive index $\Delta n^{\prime \prime}$ can be calculated using the relation $\Delta n^{\prime \prime}=\frac{\Delta \alpha \lambda}{4 \pi}$, and the real part of the refractive index can be determined from the KramersKronig relation,

$$
\Delta n^{\prime}=\frac{c_{0} \hbar}{\pi q_{e}} \mathrm{P} \int_{0}^{\infty} \frac{\Delta \alpha\left(E^{\prime}\right)}{E^{\prime 2}-E^{2}} d E^{\prime},
$$

where $c_{0}$ is the speed of light in vacuum, $\hbar$ is the reduced Planck's constant, $E$ is the photon energy, and $q_{e}$ is the fundamental electron charge [24]. $\mathrm{P}$ denotes the Cauchy principal value of the integral.

The band-gap shrinkage effect describes the phenomena where high concentrations of electrons and holes in a semiconductor material cause a decrease in the band-gap energy due to electron-electron and hole-hole interactions [24]. The amount of band-gap shrinkage $\Delta E_{g}$ caused by a carrier concentration $N$ can be approximated by an analytical equation proposed by Wolff [24-26],

$$
\Delta E_{g}=-\frac{e}{2 \pi \epsilon_{0} \epsilon_{s}}\left(\frac{3}{\pi}\right)^{\frac{1}{3}} N^{\frac{1}{3}},
$$

where $\epsilon_{0}$ is the vacuum permittivity and $\epsilon_{\mathrm{s}}(23.3$ [22]) is the static relative permittivity of the semiconductor material. To account for the band-gap shrinkage caused by the initial carrier concentration before photoexcitation $N_{0}$, the equation above is modified and the equation describing the additional band-gap shrinkage only becomes

$$
\Delta E_{g}=-\frac{e}{2 \pi \epsilon_{0} \epsilon_{s}}\left(\frac{3}{\pi}\right)^{\frac{1}{3}}\left[\left(N+N_{0}\right)^{\frac{1}{3}}-N_{0}^{\frac{1}{3}}\right] .
$$

An approximation of the change in the absorption coefficient can be determined by simply shifting the measured absorption profile by an energy equal to the change in the 

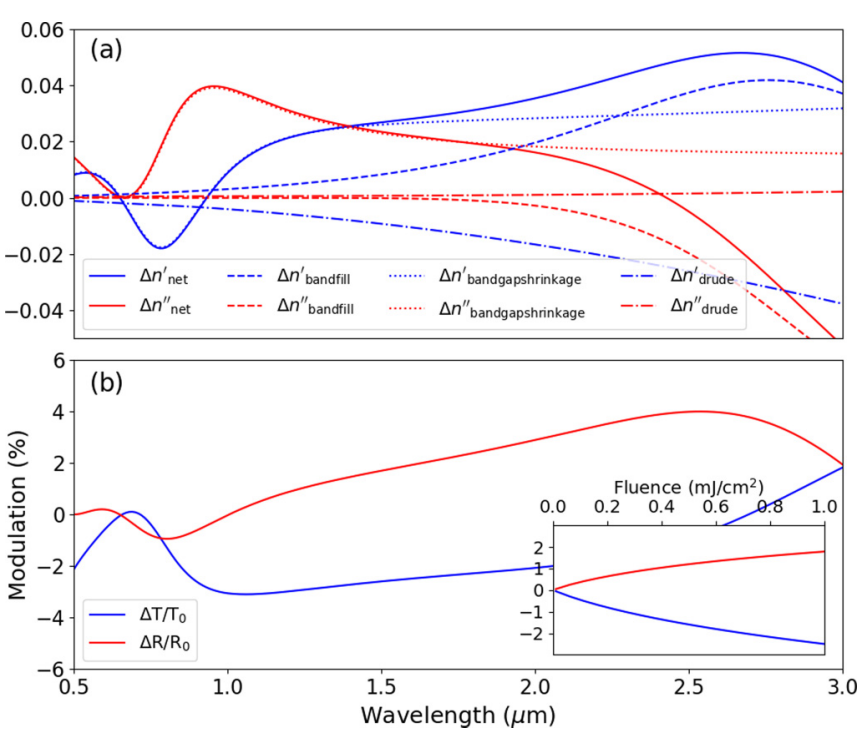

FIG. 4. (a) Change in the real (blue) and imaginary (red) refractive indices of $\mathrm{NbO}_{2}$ induced by photoexcited carriers for a $1 \mathrm{~mJ} / \mathrm{cm}^{2}$ pump pulse, caused by the Burstein-Moss effect (dashed), the bandgap shrinkage effect (dotted), and the free carrier effect (dotted dashed). (b) Modulation in the transmittance (blue) and reflectance (red) resulting from the refractive index change shown in (b). Inset: Change in $1.55-\mu \mathrm{m}$ transmittance and reflectance modulation with fluence.

band-gap energy [27], giving

$$
\Delta \alpha(E)=\alpha_{0}\left(E-\Delta E_{g}\right)-\alpha_{0}(E) .
$$

Using the same method used for the Burstein-Moss effect, the $\Delta n^{\prime}$ and $\Delta n^{\prime \prime}$ for the band-gap shrinkage effect were calculated.

The last effect of interest is the free carrier absorption effect. Excited carriers in a semiconductor act as free carriers with a corresponding Drude response that alters the semiconductor's optical properties [23]. The change in the relative permittivity due to a concentration $N$ of photoexcited carriers is described by the Drude-Lorentz model [28],

$$
\Delta \epsilon_{r}=-\frac{\frac{N q_{e}^{2}}{\epsilon_{0} m^{*}}}{\omega^{2}+i \omega \gamma},
$$

where $m^{*}$ is the electron effective mass, $\gamma$ is the damping frequency, and $\omega$ is the frequency of light in $\mathrm{rad} / \mathrm{s}$. Using a second-order Taylor expansion, the change in the complex refractive index $\Delta n$ can be calculated as

$$
\Delta n=\frac{\Delta \epsilon_{r}}{2 n_{0}}-\frac{\Delta \epsilon_{r}^{2}}{8 n_{0}^{3}},
$$

where $n_{0}$ is the original refractive index.

The resulting $\Delta n^{\prime}$ and $\Delta n^{\prime \prime}$ for the three effects discussed above are shown in Fig. 4(a). Over the majority of the visible and near-infrared (NIR) wavelength range, the band-gap shrinkage effect dominates the photoexcited carrier-induced refractive index change. At the probe wavelength of $1.55 \mu \mathrm{m}$, an overall increase in both the real and imaginary parts of the refractive index is observed. Figure 4(b) shows the expected differential transmittance based on the refractive index changes for a $1 \mathrm{~mJ} / \mathrm{cm}^{2}$ pump pulse over the visible and NIR wavelength range. Details of the calculation are given in the Supplemental Material [21]. The expected differential transmittance at $1.55 \mu \mathrm{m}$ shown in Fig. 4(b) $(-2.54 \%)$ and the measured $T_{\min }$ at $1 \mathrm{~mJ} / \mathrm{cm}^{2}$ in Fig. $2(-2.99 \%)$ are very similar. Another important observation is that the differential reflectance becomes negative over the range of approximately $650 \mathrm{~nm}$ to $1 \mu \mathrm{m}$, which matches the decrease in the reflectance at $800 \mathrm{~nm}$ observed by Beebe et al. [19]. Despite the agreement in sign between the predicted differential reflectance at $1.55 \mu \mathrm{m}$ and experimental measurements shown in Fig. 1, the predicted differential reflectance is clearly overestimated. This is likely due to the assumption that the band-gap shrinkage causes a uniform shift in the absorptivity curve at all wavelengths, whereas the effect of the bandgap shrinkage effect on the band structure is weaker further from the band-gap edge [29]. The agreement between the value at $T_{\min }$ and the predicted modulation based on photoexcited carrier-induced refractive index changes provides strong evidence that the latter is the origin of the shortlived modulation. It also explains why a ps time constant was observed for the recovery of the short-lived modulation, as electrons and holes are expected to recombine on a ps timescale.

To further support the above conclusion, the scaling of the predicted modulation with pump fluence was also examined [Fig. 4(b) inset]. As expected, at $1.55 \mu \mathrm{m}$ a differential reflectance increasing with pump fluence and a differential transmittance decreasing with pump fluence is observed. The magnitude of the differential transmittance for different pump fluences is also very similar to that observed in Fig. 2; however, unlike the measured differential transmittance, the predicted differential transmittance scales sublinearly with pump fluence. Since the initial carrier concentration $N_{0}$ was relatively high, though, the deviation from linearity in the range of $0-1 \mathrm{~mJ} / \mathrm{cm}^{2}$ is only very slight. Furthermore, a more accurate calculation of $\Delta E_{g}$ would include higher-order terms [30], and this would improve the linearity.

The last feature of interest is the ultrafast modulation. Here, the ultrafast modulation at $1.55 \mu \mathrm{m}$ was found to correspond to a decrease in both the transmittance and reflectance of $\mathrm{NbO}_{2}$. Only an increase in the imaginary part of the refractive index with no or a minimal change in the real part of the refractive index will result in this behavior. In both prior studies of $\mathrm{NbO}_{2}$ 's photoinduced modulation, the relaxation of the ultrafast modulation was attributed to the relaxation of the photoexcited carriers from their initial nonthermal distribution to a quasithermal distribution through electron-electron and electron-phonon scattering [19,20]. In materials that show this kind of effect, the strengths of the band filling and free carrier absorption effects are modified due to the change in the effective masses of the photoexcited carriers as they relax, and this results in a change in the modulation [31]. However, from the modeling shown in Fig. 4, the band filling effect should cause a decrease in absorption, and the free carrier absorption effect a large change in the real part of $\mathrm{NbO}_{2}$ 's refractive index, and therefore neither of these effects can explain the ultrafast modulation observed. Another possibility is that the ultrafast modulation represents the increase in absorption caused by two-photon absorption of the pump and 
probe pulse. This would also explain why the recovery time constant of the ultrafast modulation was limited by the laser pulse width in both this study and the one performed by Beebe et al. [19].

\section{CONCLUSION}

The results here show that the long-lived and short-lived modulation of $\mathrm{NbO}_{2}$ after excitation by a femtosecond laser seen at low pump fluences are not the result of a photoinduced IMT. Instead, the long-lived modulation was found to be caused by photothermally induced changes in the lattice temperature of $\mathrm{NbO}_{2}$, and the short-lived modulation was determined to be caused by photoexcited carrier-induced changes in the refractive index of $\mathrm{NbO}_{2}$. The ultrafast modulation is also likely to be related to two-photon absorption.

Despite the short-lived modulation not representing a purely electronic IMT, the large modulation observed is very promising for the development of nanoscale all-optical modulators. Given the photothermal nature of the long-lived modulation, it can be significantly reduced by using a longerwavelength pump pulse to reduce the excess above band-gap energy as well as using ultrathin and nanostructured $\mathrm{NbO}_{2}$ to increase the rate of heat dissipation. This has the potential to enable $\mathrm{NbO}_{2}$ based all-optical devices with speeds as high as 100 s of $\mathrm{GHz}$.

\section{ACKNOWLEDGMENTS}

The authors would like to extend our grateful appreciation to Prof. Makoto Kuwata-Gonokami, Prof. Junji Yumoto, and Dr. Kuniaki Konishi from School of Science, The University of Tokyo for important discussions and technical support. This work was supported in part by the Japan Society for the Promotion of Science (JSPS) KAKENHI under Grants No. 17K18867, No. 17H03229, No. 19J13644, and No. $18 \mathrm{H} 01468$.
[1] Z. Chai, X. Hu, F. Wang, X. Niu, J. Xie, and Q. Gong, Adv. Opt. Mater. 5, 1600665 (2017).

[2] V. R. Almeida, C. A. Barrios, R. R. Panepucci, and M. Lipson, Nature (London) 431, 1081 (2004).

[3] M. F. Yanik, S. Fan, and M. Soljacic, Appl. Phys. Lett. 83, 2739 (2003).

[4] S. Combrié, Q. V. Tran, A. De Rossi, C. Husko, and P. Colman, Appl. Phys. Lett. 95, 221108 (2009).

[5] W. Li, B. Chen, C. Meng, W. Fang, Y. Xiao, X. Li, Z. Hu, Y. $\mathrm{Xu}$, L. Tong, H. Wang, W. Liu, J. Bao, and Y. R. Shen, Nano Lett. 14, 955 (2014).

[6] A. Zylbersztejn and N. F. Mott, Phys. Rev. B 11, 4383 (1975).

[7] V. Eyert, Europhys. Lett. 58, 851 (2002).

[8] A. Joushaghani, J. Jeong, S. Paradis, D. Alain, J. S. Aitchison, and J. K. S. Poon, Opt. Express 23, 3657 (2015).

[9] P. Markov, R. E. Marvel, H. J. Conley, K. J. Miller, R. F. Haglund, and S. M. Weiss, ACS Photonics 2, 1175 (2015).

[10] K. J. Miller, K. A. Hallman, R. F. Haglund, and S. M. Weiss, Opt. Express 25, 26527 (2017).

[11] S. Wall, L. Foglia, D. Wegkamp, K. Appavoo, J. Nag, R. F. Haglund, J. Stahler, and M. Wolf, Phys. Rev. B 87, 115126 (2013).

[12] M. van Veenendaal, Phys. Rev. B 87, 235118 (2013).

[13] Z. Tao, T.-R. T. Han, S. D. Mahanti, P. M. Duxbury, F. Yuan, C.-Y. Ruan, K. Wang, and J. Wu, Phys. Rev. Lett. 109, 166406 (2012).

[14] D. Wegkamp, M. Herzog, L. Xian, M. Gatti, P. Cudazzo, C. L. McGahan, R. E. Marvel, R. F. Haglund, A. Rubio, M. Wolf, and J. Stähler, Phys. Rev. Lett. 113, 216401 (2014).

[15] A. Cavalleri, C. Toth, C. W. Siders, J. A. Squier, F. Raksi, P. Forget, and J. C. Kieffer, Phys. Rev. Lett. 87, 237401 (2001).

[16] C. Kubler, H. Ehrke, R. Huber, R. Lopez, A. Halabica, R. F. Haglund, and A. Leitenstorfer, Phys. Rev. Lett. 99, 116401 (2007).
[17] V. R. Morrison, R. P. Chatelain, K. L. Tiwari, A. Hendaoui, A. Bruhács, M. Chaker, and B. J. Siwick, Science 346, 445 (2014).

[18] D. Wegkamp and J. Stähler, Prog. Surf. Sci. 90, 464 (2015).

[19] M. R. Beebe, J. M. Klopf, Y. Wang, S. Kittiwatanakul, J. Lu, S. A. Wolf, and R. A. Lukaszew, Opt. Mater. Express 7, 213 (2017).

[20] R. Rana, J. M. Klopf, J. Grenzer, H. Schneider, M. Helm, and A. Pashkin, Phys. Rev. B 99, 041102(R) (2019).

[21] See Supplemental Material at http://link.aps.org/supplemental/ 10.1103/PhysRevResearch.1.033168 for further details on sample characterization, the pump-probe spectroscopy setup, and the derivation of the theoretical model described in this paper.

[22] Y. Sakai, N. Tsuda, and T. Sakata, J. Phys. Soc. Jpn. 54, 1514 (1985).

[23] K. T. Jacob, C. Shekhar, M. Vinay, and Y. Waseda, J. Chem. Eng. Data 55, 4854 (2010).

[24] B. R. Bennett, R. A. Soref, and J. A. Del Alamo, IEEE J. Quantum Electron. 26, 113 (1990).

[25] P. A. Wolff, Phys. Rev. 126, 405 (1962).

[26] D. Auvergne, J. Camassel, and H. Mathieu, Phys. Rev. B 11, 2251 (1975).

[27] P. A. Danilov, A. A. Ionin, S. I. Kudryashov, S. V. Makarov, A. A. Rudenko, P. N. Saltuganov, L. V. Seleznev, V. I. Yurovskikh, D. A. Zayarny, and T. Apostolova, J. Exp. Theor. Phys. 120, 946 (2015).

[28] J. D. Jackson, Classical Electrodynamics, 3rd ed. (Wiley, New York, 1999).

[29] H. Kalt and M. Rinker, Phys. Rev. B 45, 1139 (1992).

[30] J. Kumar and A. Kumar Srivastava, J. Appl. Phys. 115, 134904 (2014).

[31] A. J. Sabbah and D. M. Riffe, Phys. Rev. B 66, 165217 (2002). 